\title{
PACKET ERROR RATE AND EFFICIENCY CLOSED-FORM EXPRESSIONS FOR CROSS-LAYER HYBRID ARQ SCHEMES
}

\author{
A. Le Duc, C. J. Le Martret \\ Thales Communications, Colombes, France \\ aude.leduc@fr.thalesgroup.com \\ christophe.le_martret@fr.thalesgroup.com
}

\author{
P. Ciblat \\ TELECOM ParisTech, Paris, France \\ philippe.ciblat@enst.fr
}

\begin{abstract}
For several years, HARQ schemes have been extensively analysed in terms of Packet Error Rate and Efficiency at the MAC level. Nevertheless, in order to get realistic performance of the whole system, performance analysis at the IP level is crucial. Therefore, we derive in closed-form expressions the performance (Packet Error Rate and Efficiency) of any HARQ scheme (ARQ, Incremental Redundancy HARQ, Chase Combining) at the IP level. Moreover cross-layer optimization strategy between MAC and IP layers for HARQ schemes developed initially by [9] for ARQ schemes is considered.
\end{abstract}

\section{INTRODUCTION}

Different mechanisms of coding may be carried out in a communication system. One of the most popular is the Hybrid-ARQ (HARQ) which combines ARQ schemes based on packet retransmission and forward error coding based on error corrections. Various HARQ schemes have been proposed in the literature: one can mention Chase-Combining (CC) HARQ ([1]) and Incremental Redundancy (IR) HARQ ([2, 3, 4]). Theoretical analysis of HARQ schemes through Packet Error Rate (PER), efficiency, and delay is a crucial task since it enables to provide practical insights about HARQ schemes design.

Usually the theoretical or practical comparisons of different HARQ schemes are only done at Radio Access layer and the considered packets are the so-called MAC packets. In such a context, there is an important number of papers focused on HARQ performance analysis. Regarding the basic ARQ schemes, [5] has established closed-form expressions of the PER and efficiency at the MAC level. Extensions of these expressions to HARQ schemes can be found in $[6,7]$ for Packet Error Rate and in [8] for efficiency.

Recently the authors in [9] propose to analyse and to optimize ARQ schemes at the IP level by taking into account the fact that the MAC packets belong to a same packet coming from the network layer. Indeed the network layer packets (also called IP packets) are fragmented in order to have the length of the MAC packets required by the radio access layer. As an optimization, the authors suggested to transfer the number of retransmission per MAC packet into a global transmission credit associated with the IP packet in the context of ARQ scheme. Theoretical expressions for packet error rate and delay are given in [9] for ARQ schemes at the IP level with and without taking into account the suggested optimization. Notice that an extension of the cross-layer strategy proposed in [9] to HARQ schemes is straightforward and introduced later in the paper.

In this paper, we are interesting in the performance evaluation of any HARQ scheme in terms of packet error rate and efficiency (defined as the ratio between received information bits with no error and transmitted bits) at the IP level. Our contribution is to provide closed-form expressions for these two metrics in the cross-layer and conventional strategies at the IP level.

The paper is organized as follows: in Section 2, we describe the communication scheme. In Section 3, we derive the closedform expressions of the considered metrics. Section 4 is devoted to numerical illustrations. Conclusion is drawn in Section 5.

\section{COMMUNICATION SCHEME DESCRIPTION}

\subsection{Layer Model}

In our system, we consider only the three first ISO layers : layer 1 (also called physical/PHY layer), layer 2 (also called radio access/data link/MAC layer) and layer 3 (also called network/IP layer). We use the conventional naming of Service Data Unit (SDU) and Packet Data Unit (PDU) which is specified according to the layer [11]. For instance for layer 2, we use DSDU and DPDU.

At the transmitter, layer 2 gets DSDU packets (also called IP packets) of length $L_{\mathrm{IP}}$ from layer 3 . DSDU packet is fragmented into $N$ fragments (also called MAC packets) of length $L_{\mathrm{MAC}}=L_{\mathrm{IP}} / N$. From these MAC packets, DPDU packets are generated and transmitted to layer 1 . The way DPDUs are built from MAC packets depends upon the retransmission scheme and will be detailed later on. We assume that each DPDU packet has the same length $L_{P} \geq L_{\mathrm{MAC}}$. These DPDU packets are finally sent through the propagation channel into a frame structure. In order to focus on retransmission performance only, we assume a single user case, that there is no queuing and that MAC layer sends one DPDU per frame on the PHY layer. After propagating through the channel, the receive packet is demodulated (and decoded if necessary) and the DPDU packet is sent to the layer 2 which decides ACKnowledgment (ACK) or a Negative ACKnowledgment (NACK) back to the transmitter accordingly. Then the delivered fragmented MAC packets obtained from DPDU packets enable layer 2 to reconstitute DSDU packets that are sent to layer 3.

\subsection{Retransmission Schemes}

In order to characterize each ARQ/HARQ scheme, we actually can distinguish the retransmission mechanism (RM) that focuses 
on the operation done at the transmitter side, from the receiver processing (RP) that is performed at the receiver side. The global retransmission scheme is a concatenation of both.

\subsubsection{Retransmission Mechanisms}

The RM explains the transmitter behavior when continuously receiving NACKs. For all the RMs, an header is systematically added to the incoming fragment, followed by a CRC encoding in order to check the packet integrity at the receiver side.

RM1: ARQ. The DPDU is simply constituted by the fragmented MAC packet with the header and CRC. The transmitter may retransmit the same DPDU at most $P_{x}=P+1$ times. $P$ is the so-called persistence.

RM2: HARQ of Type I. The DPDU is constituted by the fragmented MAC packet with the header and CRC that is then encoded by a Channel Coding of rate $R=1 / r$. The transmitter retransmits the same coded DPDU at most $P_{x}=P+1$ times.

RM3: HARQ of Type II (with Incremental Redundancy). The fragmented MAC packet with the header and the CRC is encoded by a Channel Coding of rate $R_{0}=1 / r_{0}$ (known as mother code). The redundancy bits are then broken up into $\left(r_{0}-1\right)$ subblocks of same length $L_{P}$ and transformed into a set of DPDUs numbered as $\{\operatorname{DPDU}(i)\}_{i=1}^{r_{0}}$. DPDU(1) corresponds to the information bits whereas other DPDU $(i)$ (for $i=2, \cdots, r_{0}$ ) correspond to redundancy. The transmitter starts to transmit DPDU(1), then DPDU(2), till DPDU $\left(r_{0}\right)$. As the persistence is given per fragment, there are at most $P_{x}=r_{0}(P+1)$ successive packet transmissions.

\subsubsection{Receiver Processing}

The RP depicts the way the incoming DPDUs are processed in order to decide if the estimated fragmented MAC packet is corrupted or not. In the sequel, a packet is "received" when no error occurs.

RP1: Packet by packet. The receiver treats the DPDU packets one by one without memory.

RP2: Flush memory. It consists in processing sequentially the incoming packets: checking the CRC for DPDU(1), then decoding the rate $1 / 2$ after receiving DPDU(2) and checking the CRC, till reception of DPDU $\left(r_{0}\right)$ which is decoded with mother code of rate $R_{0}$ followed by the CRC checking. Then, if the fragmented MAC packet is not received after the DPDU $\left(r_{0}\right)$ decoding and the persistence is not reached, the received packet memory is flushed (put to zero) and the process starts again. This is applicable to RM3 (i.e., IR-HARQ).

RP3: Chase Combining. The receiver combines the incoming DPDU packets using the Chase Combining algorithm [1]. RP3 is applicable to the three RMs as soon as $P>0$.

\subsection{Cross-layer Strategies}

The conventional retransmission schemes are usually applied at the MAC level. Recently, based on the fact that if one DPDU at layer 2 is missing at the receiver side the corresponding DSDU at layer 3 is dropped, the authors in [9] proposed to enhance the ARQ scheme by granting a global retransmission credit, noted $C$, to the set of fragmented MAC packets belonging to the same IP packet. Thus, rather than allowing each of the $N$ fragments (belonging to the same DSDU) to be retransmitted $P$ times, the new scheme allocates $C$ transmissions to the set of $N$ fragments. This strategy at layer 2 actually takes into account the fact that layer 3 is expecting complete packets and constitutes a cross-layer optimization of the link control. In the later we will refer the conventional one to as PDU-Based Strategy (PBS) and the cross-layer one to as SDUBased Strategy (SBS). Extending the strategy developed for ARQ scheme in [9] to the HARQ (RM2, RM3 or any other HARQ) is straightforward by using an HARQ transmission scheme per fragment instead of the ARQ one.

\section{PERFORMANCE CLOSED-FORM EXPRESSIONS}

The performance evaluation of the retransmission schemes is usually assessed through metrics such as packet error rate, efficiency, etc. The main contribution of this paper is to give general closedform expression of those metrics for cross-layer optimized HARQ and standard HARQ at the IP level.

\subsection{Notations and Preliminaries}

For PBS, all the metrics can be considered at the MAC level and at the IP level, whereas for SBS, only IP level makes sense. For the notation, we will put the subscript 'MAC' for MAC level and 'IP' for the IP level. Depending on the retransmission strategy (PBS or SBS), we will differentiate the metrics by adding an upper script, respectively with a ' $P$ ' or a ' $S$ '. We also assume without loss of generality that the ACK/NACK transmissions are delay-free.

The closed-form expressions we derive in this section are functions of all the parameters $(P, N, C)$ and the elementary packet error rate $\pi_{j}$ defined as the PER corresponding to the $(j+1)$ th DPDU transmission when the $j$ previous DPDU transmissions have failed. Those packet error rates are computed only one time (by simulation or using analytical approaches) and will serve to calculate all the performance using the analytical expressions.

We derive the metric closed-form expressions in three steps. First we derive expressions using the probability $p_{n}^{x}(k)$ which is defined as the probability that $n$ successive fragmented MAC packets are successfully received in exactly $k$ DPDU transmissions for xBS. The obtained expressions derived for both PBS and SBS are valid whatever the RM and RP. The second step consists in expressing $p_{n}^{x}(k)$ as a function of the elementary probability $p_{1}(k)$ that is independent of the cross-layer strategy. In the last step, we derive the $p_{1}(k)$ expressions as a function of the $\pi_{j}$. Finally even simplified novel expressions for ARQ schemes at IP level are proposed.

\subsection{Metrics Expressions vs. $p_{n}^{x}(k)$}

\subsubsection{Packet Error Rate}

The packet error rate, denoted by $\Pi$, is defined as the ratio between the number of receive packets and the number of transmit packets. At the MAC level, the PER is easily obtained in the PBS context since the probability that a fragmented MAC packet is correctly received is equal to the probability that the fragment is received in one transmission, or two transmissions, till the maximum number of transmission. Thus, from the assumptions and the $p_{n}^{x}(k)$ definition, we obtain the well-known result

$$
\Pi_{\mathrm{MAC}}^{P}=1-\sum_{k=1}^{P_{x}} p_{1}(k),
$$


where $P_{x}$ is the maximum number of transmit DPDUs that takes different expressions depending on the RM (see Section 2.2.1).

At the IP level, since the fragment transmissions are independent, it is straightforward to obtain

$$
\Pi_{\mathrm{IP}}^{P}=1-\left(1-\Pi_{\mathrm{MAC}}^{P}\right)^{N} .
$$

In the SBS case, we have to consider the global credit of transmission $C$ per DSDU, thus there is only $\Pi_{\mathrm{IP}}^{S}$ to consider. Using the same derivation as previously, we need to express the probability that a DSDU is received correctly. This probability is equal to the probability that the DSDU is received after $N$ transmit DPDUs, or after $(N+1)$ DPDUs, till the maximum number of transmissions. We thus obtain

$$
\Pi_{\mathrm{IP}}^{S}=1-\sum_{k=N}^{C} p_{N}^{S}(k)
$$

\subsubsection{Efficiency}

The efficiency, denoted by $\eta$, is defined as the ratio between the number of received information bits with no error and the number of transmit bits. At the IP level, we can derive a single expression for both PBS and SBS. The efficiency can be easily obtained as

$$
\eta_{\mathrm{IP}}^{x}=N \cdot \frac{\rho}{\bar{n}_{\mathrm{IP}}^{x}}
$$

where $\rho:=L_{\mathrm{MAC}} / L_{P}$ is the proportion of information bits in one fragmented MAC packet and $\bar{n}_{\mathrm{IP}}^{x}$ is the mean number of transmit DPDUs between two successive receive DSDUs for xBS. This quantity can thus be expressed by definition as:

$$
\bar{n}_{\mathrm{IP}}^{x}=\sum_{i=1}^{+\infty} \bar{n}_{\mathrm{IP}}^{x}(i) \cdot \operatorname{Pr}\{\text { DSDU received after } i \text { transmit DSDUs }\}
$$

where $\bar{n}_{\mathrm{IP}}^{x}(i)$ is the mean number of transmit DPDUs when a DSDU is received after $i$ trials. This expression takes into account the fact that between two successive receive DSDUs, some transmit DSDUs may have been dropped and that the transmitter may have transmit one DSDU, or two DSDUs, till infinity. The probability that a DSDU is received after $i$ transmit DSDUs is easily equal to $\left(1-\Pi_{\mathrm{IP}}^{x}\right)\left(\Pi_{\mathrm{IP}}^{x}\right)^{(i-1)}$. When a DSDU is received after $i$ trials, it corresponds to the one successful trial along with $(i-1)$ unsuccessful trials. Thus, $\bar{n}_{\mathrm{IP}}^{x}(i)$ can be expressed as:

$$
\bar{n}_{\mathrm{IP}}^{x}(i)=\bar{n}_{\mathrm{IP}}^{x}(1)+(i-1) \cdot \breve{n}_{\mathrm{IP}}^{x}
$$

where $\breve{n}_{\mathrm{IP}}^{x}$ is the mean number of transmit DPDUs when a DSDU fails to be received. Then, putting (6) into (5) yields to

$$
\eta_{\mathrm{IP}}^{x}=\frac{\rho \cdot N \cdot\left(1-\Pi_{\mathrm{IP}}^{x}\right)}{\breve{n}_{\mathrm{IP}}^{x} \cdot \Pi_{\mathrm{IP}}^{x}+\bar{n}_{\mathrm{IP}}^{x}(1) \cdot\left(1-\Pi_{\mathrm{IP}}^{x}\right)} .
$$

To proceed further, we need now to specify the expression of $\breve{n}_{\mathrm{IP}}^{x}$ and $\bar{n}_{\mathrm{IP}}^{x}(1)$ according to the retransmission strategy. For PBS, we can easily identify that

$$
\bar{n}_{\mathrm{IP}}^{P}(1)=N\left(1-\Pi_{\mathrm{MAC}}^{P}\right)^{-1} \sum_{k=1}^{P_{x}} k \cdot p_{1}(k)
$$

where the fragmented MAC packets transmissions are assumed independent. As for $\breve{n}_{\mathrm{IP}}^{P}$, a DSDU not received means that at least one fragmented MAC packet has been dropped, thus there are at least $P_{x}$ transmissions. For the remaining $(N-1)$ transmissions, they can be successful or not. Thus we can write $\breve{n}_{\mathrm{IP}}^{P}=$ $P_{x}+(N-1) \cdot \bar{n}$, where $\bar{n}$ is the mean number of transmit DPDUs per fragmented MAC packet, whatever this MAC packet is received or not. One way to derive this quantity is to list all the possible events. First we count the different successful events (from 1 transmission to $P_{x}$ ) given by $\sum_{k=1}^{P_{x}} k \cdot p_{1}(k)$ and then we have to add the unsuccessful event (the $P_{x}$ transmissions fail) that is given by $P_{x} \cdot \Pi_{\mathrm{MAC}}^{P}$ which leads to $\bar{n}=\sum_{k=1}^{P_{x}} k \cdot p_{1}(k)+P_{x} \cdot \Pi_{\mathrm{MAC}}^{P}$. We finally come to the following expression:

$$
\breve{n}_{\mathrm{IP}}^{P}=P_{x}+(N-1) \cdot\left(\sum_{k=1}^{P_{x}} k \cdot p_{1}(k)+P_{x} \cdot \Pi_{\mathrm{MAC}}^{P}\right) .
$$

For SBS, we simply have

$$
\breve{n}_{\mathrm{IP}}^{S}=C
$$

since the DSDU is not received if the maximum number of transmissions per DSDU equal to $C$ is reached. Moreover, one can check that

$$
\bar{n}_{\mathrm{IP}}^{S}(1)=\left(1-\Pi_{\mathrm{IP}}^{S}\right)^{-1} \sum_{k=N}^{C} k \cdot p_{N}^{S}(k) .
$$

At the MAC level for PBS, the result can be obtained by putting $N=1$ in PBS at the IP level or by putting $N=1$ and $C=P_{x}$ in SBS. Then we have

$$
\eta_{\mathrm{MAC}}^{P}=\frac{\sum_{k=0}^{P_{x}} p_{1}(k)}{P_{x}\left(1-\sum_{k=0}^{P_{x}} p_{1}(k)\right)+\sum_{k=0}^{P_{x}} k p_{1}(k)}
$$

which is identical to the equation provided in [8].

All the metrics related to the PBS case involve only $p_{1}(k)$ whereas the metrics associated with SBS case depend on $p_{n}(k)$ which is expressed in terms of $p_{1}(k)$ in the next subsection.

3.3. $p_{n}^{x}(k)$ vs. $p_{1}(k)$

In this subsection, we find that $p_{n}^{S}(k)$ can be expressed as a function of the $p_{1}(k)$ probabilities. The idea is to remark that the different events related to $p_{n}^{S}(k)$ are constituted by $n$ successive independent successful transmissions of one DPDU, and thus can be written as:

$$
p_{n}^{S}(k)=\sum_{\underline{\mathbf{q}} \in Q_{k, n}^{S}} p_{1}\left(q_{1}\right) p_{1}\left(q_{2}\right) \cdots p_{1}\left(q_{n}\right)
$$

where $\underline{\mathbf{q}}:=\left(q_{1}, q_{2}, \cdots, q_{n}\right)$ and where the summation set $Q_{k, n}^{S}=$ $\left\{\left(q_{1}, q_{2}, \cdots, q_{n}\right) \mid \sum_{i=1}^{n} q_{i}=k, 0<q_{i}\right\}$ takes into account the fact that the $n$ packets are received in exactly $k$ transmissions. Although Eq. (12) provides $p_{n}^{S}(k)$ with respect to $p_{1}(k)$, it is useless in practice since the determination of the set $Q_{k, n}^{S}$ needs an exhaustive search which is not tractable when $n$ and $k$ become large. As remarked in [9] (with a simpler set $Q$ than ours), the Buzen's algorithm [12] enables us to calculate recursively Eq. (12) as follows

$$
p_{n}^{S}(k)=\sum_{k^{\prime}=1}^{k-n+1} p_{1}\left(k^{\prime}\right) p_{n-1}^{S}\left(k-k^{\prime}\right) .
$$


3.4. $p_{1}(k)$ vs. $\pi_{j}$

Whatever the retransmission scheme, $p_{1}(k)$ is the probability that one fragmented MAC packet is received correctly after $k$ DPDU transmissions. It can be thus taken the following generic form

$$
p_{1}(k)=\left(1-\pi_{k-1}\right) \cdot \prod_{i=0}^{k-2} \pi_{i} .
$$

First of all, given previous generic expressions for PER and efficiency in cross-layer (' $S$ ') or standard (' $P$ ') strategy, we now are able to evaluate the performance of any HARQ scheme without simulating it at the IP level. The time needed to evaluate the performance lyes only on the execution time of the term $\pi_{j}$. We remind that these terms only depend on noise level and feedforward error coding scheme.

Secondly, notice that, to our best knowledge, one of the novelty of this paper is to provide exact closed-form expressions for PER and efficiency even in HARQ schemes in standard PBS context (i.e., without cross-layer) at the IP level.

Thirdly, these derivations enable to analyze some links between different schemes. For instance, as $p_{n}^{P}(k)$ can be written similarly to Eq. (12) by replacing $Q_{k, n}^{S}$ with $Q_{k, n}^{P}=\left\{\left(q_{1}, q_{2}, \cdots\right.\right.$, $\left.\left.q_{n}\right) \mid \sum_{i=1}^{n} q_{i}=k, 0<q_{i} \leq P_{x}\right\}$, one can show that if $C \geq$ $N P_{x}$, the SBS approach is always better than the PBS one in terms of PER whatever the retransmission scheme (RM and RP).

\subsection{Simplified Performance Closed-Form Expressions for ARQ}

In the previous section, we derived PER and efficiency as a function of $\pi_{j}$. Those expressions cannot be simplified further, except for the ARQ scheme (RM1-RP1). In such a case, $\forall j, \pi_{j}=\pi_{0}$ and more compact closed-form expressions for the considered metrics can be found for both PBS and SBS cases at the IP level.

In the ARQ schemes, Eq. (13) takes the simple form:

$$
p_{1}(k)=\left(1-\pi_{0}\right) \pi_{0}^{(k-1)} .
$$

We firstly focus on the Packet Error Rate evaluation. For PBS at the IP level, putting Eq. (14) into Eq. (1), and then Eq. (1) into Eq. (2) leads to

$$
\Pi_{\mathrm{IP}}^{P}=1-\left(1-\pi_{0}^{(P+1)}\right)^{N} .
$$

For SBS at IP level, putting Eq. (14) into Eq. (12), and then Eq. (12) into Eq. (3) enables the following simple expression

$$
\Pi_{\mathrm{IP}}^{S}=\frac{\pi_{0}^{(C-N+1)} \Gamma(C+1)\left(1-\pi_{0}\right)^{N} F_{C+1}^{C-N+2}\left(1, \pi_{0}\right)}{\Gamma(N)}
$$

where $\Gamma(x)$ and $F_{x}^{y}(w, z):={ }_{2} F_{1}(w, x ; y ; z)$ are the so-called gamma and hypergeometric functions respectively.

At the MAC level, $\Pi_{\mathrm{MAC}}^{P}$ is obtained by putting $N=1$ into Eq. (15) and is equal to $\pi_{0}^{P+1}$ as already mentioned in [5].

We now move on the efficiency evaluation. For PBS at the IP level, after straightforward but tedious algebraic manipulations, using Eqs. (14)-(9)-(8)-(7) leads to (17). For SBS at the IP level, using Eqs. (14)-(12)-(11)-(7) leads also to the following equation

$$
\eta_{\mathrm{IP}}^{x}=\rho \frac{A_{x}}{B_{x}+D_{x}}
$$

with

$$
\left\{\begin{aligned}
A_{P} & =N\left(\pi_{0}-1\right)\left(1-\pi_{0}^{(P+1)}\right)^{N}, \\
B_{P} & =\left((N-1) \pi_{0}^{(P+1)}+1\right)\left(\pi_{0}^{(P+1)}\right. \\
& \left.-(P+1) \pi_{0}+P\right)\left(1-\pi_{0}^{(P+1)}\right)^{(N-1)}, \\
D_{P} & =\pi_{0}^{(P+1)}(N-1)+\pi_{0}(P+1)-P-N,
\end{aligned}\right.
$$

and

$$
\left\{\begin{aligned}
A_{S} & =N\left(\pi_{0}-1\right)\left(\left(1-\pi_{0}\right)^{N} \pi_{0}^{(C+1)} \Gamma(C+1)\right. \\
& \left.\times F_{C+1}^{C-N+2}\left(1, \pi_{0}\right)-\pi_{0}^{N} \Gamma(N)\right), \\
B_{S} & =\left(1-\pi_{0}\right)^{N}\left(\pi_{0}-1\right) \pi_{0}^{(C+1)}(\Gamma(C+2) \\
& -C \Gamma(C+1)) F_{C+1}^{C-N+2}\left(1, \pi_{0}\right), \\
D_{S} & =\left(\pi_{0}^{(C+2)} \Gamma(C+2) F_{C+2}^{C-N+3}\left(2, \pi_{0}\right)+N \Gamma(N) \pi_{0}^{N}\right) \\
& \times\left(1-\pi_{0}\right)^{N}\left(\pi_{0}-1\right) .
\end{aligned}\right.
$$

Once again, $\eta_{\mathrm{MAC}}^{P}$ is obtained by putting $N=1$ into Eq. (18) and is equal to $1-\pi_{0}$ as already noticed in [5]. One can remark that, the efficiency depends on the persistence $P$ at the IP level whereas it is well-known that the efficiency in ARQ scheme at the MAC level is independent of persistence.

\section{NUMERICAL ILLUSTRATIONS}

In this simulation part, due to the lack of space, we only focus on IR-HARQ (i.e., RM3-RP2) at the IP level. The theoretical and empirical evaluations of this scheme are done under the following assumptions:

- The IR-HARQ is implemented with the Rate Compatible Punctured Convolutional (RCPC) codes ([3]) with a mother code rate of $R_{0}=1 / 4$. The number of fragments per DSDU is $N=3$. The modulation is a Binary Phase Shift Keying (BPSK) modulation.

- We consider an Additive White Gaussian Noise channel.

- The ACK/NACK feedback is error-free. The CRC is assumed to be ideal and to not add an overhead. No coding scheme is supposed to be used at the PHY layer.

Theoretical expressions of PER and efficiency are obtained by inserting the estimated values of $\pi_{j}$ (for $j \in\{0, \cdots, k-1\}$ ) in Eq. (13). Empirical PER and efficiency are obtained by sending thousand DSDUs.

In Fig. 1, we plot theoretical and empirical PER versus SNR for different values of the global transmission credit at the IP level. For SBS, this credit is equal to $C_{\mathrm{SBS}}=C$ whereas, for PBS, the global credit is fixed to $C_{\mathrm{PBS}}=N(P+1) / R_{0}$. First of all, we remark that theoretical curves perfectly match empirical ones. As mentioned at the end of Section 3.4, we here confirm that SBS offers a large performance gain compared to PBS in terms of PER for the same global retransmission credit (i.e., when $C_{\mathrm{SBS}}=$ $C_{\mathrm{PBS}}$ ). Moreover, whatever the considered strategy, the higher $C$ is, the better PER is.

In Fig. 2, we display theoretical and empirical efficiency versus SNR for different values of the global transmission credit. Once again, theoretical and empirical curves are in agreement. We show that SBS yields better performance than PBS in terms of efficiency when the same global credit is used.

In Fig. 3, we plot theoretical PER versus theoretical efficiency for different values of global credit and SNR. We remark that the global credit plays an important role in order to adjust the tradeoff between PER and efficiency. Thus, it is worthy to consider 


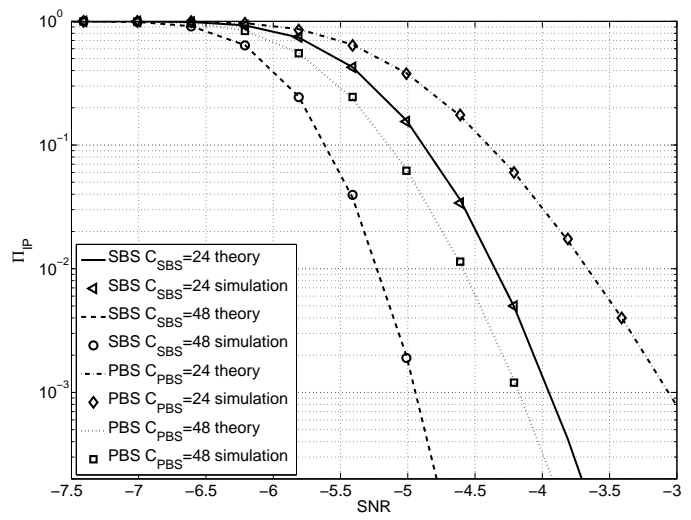

Fig. 1. Theoretical and empirical PER vs. SNR.

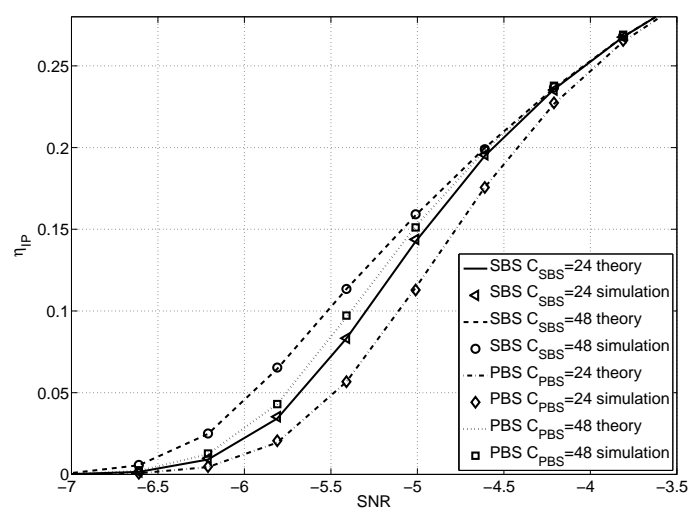

Fig. 2. Theoretical and empirical efficiency vs. SNR.

the value of the global credit $C$ for carrying out an efficient radio resource allocation management (RRM). For instance, one can select the value of $C$ (for either SBS or PBS strategy) corresponding to the best pair $\left(\Pi_{\mathrm{IP}}, \eta_{\mathrm{IP}}\right)$ that satisfies the QoS requirement of the considered system. Due to our closed-form expressions, such a RRM algorithm can be performed with a negligeable extra computation load compatible with low complex and low cost terminals.

\section{CONCLUSION}

Closed-form expressions for the PER and the efficiency of any HARQ scheme at the IP level (and not the MAC level) have been proposed in this paper. Further works may concern the theoretical evaluation of the transmission latency as well as a more precise analysis of the influence of design parameters (such as the global transmission credit, the number of fragments per DSDU). This last analysis has been omitted here due to page limitation.

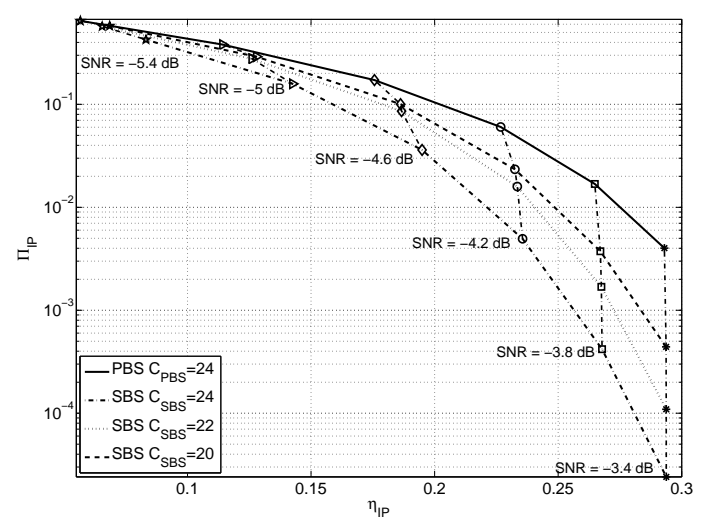

Fig. 3. Theoretical PER versus theoretical efficiency for different SNR and global transmission credit.

\section{REFERENCES}

[1] D. Chase, "Code Combining - A maximum likelihood decoding approach for combining an arbitrary number of noisy packets," in IEEE Trans. Commun., vol. 33, no. 5, pp 385393, May 1985.

[2] S. Lin and P. S. Yu, "A hybrid ARQ with parity retransmission for error control of satellite channel," in IEEE Trans. Commun., vol. 30, no. 7, pp 1701-1719, July 1982.

[3] J. Hagenauer, "Rate-Compatible Punctured Convolutional codes (RCPC codes) and their applications," in IEEE Trans. Commun., vol. 36, no. 4, pp. 389-400, April 1988.

[4] D. Mandelbaum, "An adaptive-feedback coding scheme using incremental redundancy," in IEEE Trans. Inform. Theory, vol. 20, no. 3, pp 388-389, May 1974.

[5] S. Lin and D. Costello, "Error Control Codings: fundamentals and applications," Prentice-Hall, 1983.

[6] D. Costello and S. Lin, "Error Control Coding," Pearson Higher Education, 2004.

[7] L. Badia, M. Levorato, and M. Zorzi, "Markov analysis of selective repeat type II HARQ using block codes," in IEEE Trans. Commun., vol. 56, no. 9, pp 1545-1555, Sept. 2008.

[8] S. Sesia, G. Caire, and G. Vivier, "Incremental redundancy hybrid ARQ schemes based on LDPC codes," in IEEE Trans. Commun., vol. 52, no. 8, pp. 1311-1321, Aug. 2004.

[9] Y. Choi, S. Choi, and S. Yoon, "MSDU-based ARQ Scheme for IP-Level Performance Maximization," in Proc. IEEE Globecom Conf., vol. 5, pp. 2495-2499, Dec. 2005.

[10] H. van Duuren, "Error Probability and Transmission Speed on Circuits Using Error Detection and Automatic Repetition of signals," in IRE Trans. Commun. Systems, vol. 9, no. 1, pp 38-50, Mar. 1961.

[11] A. S. Tanenbaum, "Computer Networks," Prentice Hall, 2002.

[12] J.P. Buzen, "Computational algorithms for closed queuing networks with exponential series," in Commun. ACM, vol. 16, no. 9, pp. 527-531, Sept. 1973. 